\title{
Maintaining and Monitoring the Infrastructure of the NorNeT CORE Testbed for Multi-Homed Systems
}

\author{
Thomas Dreibholz, Jarle Bjørgeengen, Jonas Werme \\ Simula Research Laboratory \\ Centre for Resilient Networks and Applications \\ Martin Linges vei 17, 1364 Fornebu, Norway \\ \{dreibh,jonasw\}@simula.no, jarle@bjorgeengen.net
}

\begin{abstract}
NORNeT CoRE is the world's first, open, largescale Internet testbed for multi-homed systems and applications. Particularly, it is currently used for research on topics like multipath transport and resilience. Researchers can run experiments on distributed, programmable nodes that are distributed over various locations and providing access to multiple different Internet service providers (ISP) with different access technologies. Clearly, a key feature of this testbed is to work in the real-world Internet. That is, it is especially desired to expose experiments to real Internet behaviour like background traffic. However, for the researcher, it is necessary to actually know how paths - being used for an experiment - actually behave: Are the paths actually working? How are the round-trip times among sites over different ISPs, etc.. How did the behaviour change over time?

To provide such information to the researchers, we have designed and developed a maintenance and monitoring infrastructure for the NORNET CORE testbed. In this paper, we will first introduce this infrastructure. Furthermore, we will demonstrate its usefulness with some useful, real-world examples. Our infrastructure has now become part of the testbed, and it is therefore available for all users of NORNET CORE as well.

Keywords: NORNET CORE, Internet Testbed, Monitoring, Multi-Homing, Path Characteristics, Resilience
\end{abstract}

\section{INTRODUCTION}

With the growing popularity of cloud applications, a steadily increasing number of users rely on ubiquitous Internet access. Without a working and stable connectivity, such applications simply become unusable. Technologies like Reliable Server Pooling (RSerPool) [1], [2] can ensure the redundancy of servers and support session failovers. In order to avoid that the Internet access becomes a single point of failure, a user can connect to multiple Internet service providers (ISP) simultaneously, which is commonly denoted as multi-homing. A Transport Layer protocol like the Stream Control Transmission Protocol (SCTP) [3] can utilise the multi-homing feature to allow endpoints to communicate, as long as at least one path in the Internet remains available. However, having to pay for multiple ISP connections frequently leads to the desire of utilising all connections simultaneously. For example, load sharing can be used to improve the payload throughput. On the Transport Layer, this is denoted as multipath transport. It is supported by SCTP with the Concurrent

\footnotetext{
${ }^{1}$ Parts of this work have been funded by the Research Council of Norway (Forskingsrådet), prosjektnummer 208798/F50.
}

Multipath Transfer for SCTP (CMT-SCTP) [4]-[6] extension. The Transmission Control Protocol (TCP) [7], with a history of more than 3 decades, supports neither multi-homing nor multipath transport. However, the Multi-Path TCP (MPTCP) [8], [9] extension adds both features. Both extensions, i.e. CMT-SCTP and MPTCP, are currently under standardisation in the IETF and "work in progress". For this purpose, research on various topics of multi-homing and multi-path transport is currently ongoing, e.g. on path stability [10], congestion control [5], [11] and fairness [12], load sharing [13]-[15], scheduling [16], [17], network coding [18], shared bottleneck detection [19], [20], and various other topics.

Clearly, research on Internet transport and applications requires evaluations in real-world Internet setups. For this purpose, the NORNET testbed for multi-homed systems is currently being built up. It provides researchers the possibility to perform research experiments on distributed, programmable nodes with access to multiple ISPs as well as IPv6 support in addition to IPv4. Of course, realistic Internet setups include effects like background traffic. It is therefore crucial for the researchers to get information on what is going on in the testbed, in order to analyse and interpret evaluation results.

In this paper, we therefore introduce our monitoring infrastructure that we have designed and developed in order to provide this information to the researchers. We will furthermore demonstrate the usefulness of this infrastructure by a realworld example.

\section{The NorNet TeStBed}

A researcher intending to run larger-scale Internet experiments with multi-homed systems is challenged with a significant amount of logistics work: remote sites have to be found to host research systems, ISP contracts have to be made, hardware has to be deployed, and maintenance activities have to be coordinated among multiple instances (at least: the researcher, the hardware vendors, each remote site and each remote site's ISPs). To make it easier to run such experiments in the Internet, the Simula Research Laboratory $[21]^{2}$ is establishing the open NORNET testbed [22] for multi-homed systems. NORNET is funded by the Research Council of Norway (Forskingsrådet) and consists of programmable research nodes distributed all

\footnotetext{
${ }^{2}$ Simula Research Laboratory: https://www.simula.no.
} 

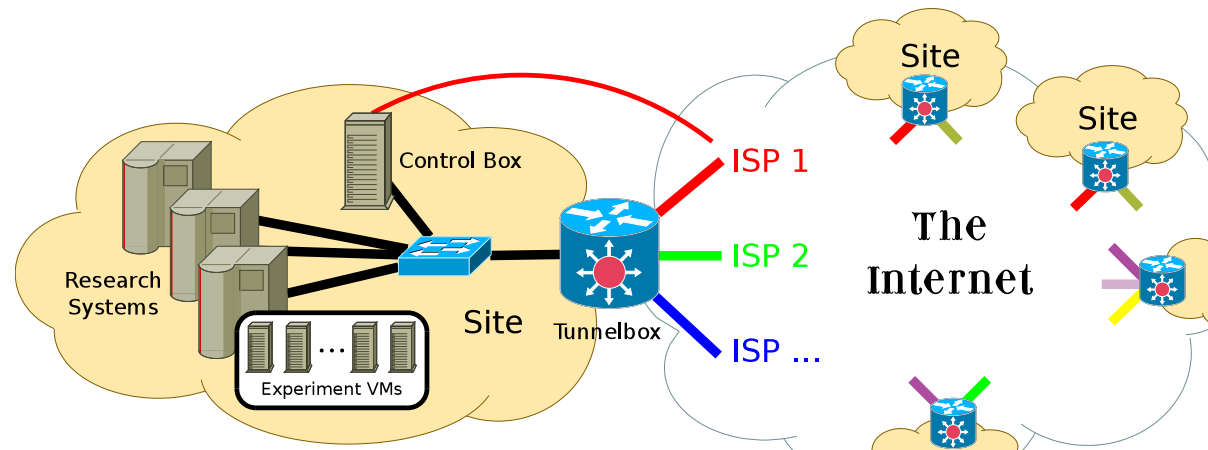

Site

Figure 1. The NORNet CORE Testbed Structure

\begin{tabular}{|c|c|c|c|c|c|}
\hline No. & Site & ISP 1 & ISP 2 & ISP 3 & ISP 4 \\
\hline 1 & Simula Research Laboratory, NO (1) & Uninett $(1)^{\mathrm{X}, 6}$ & Hafslund $(2)^{X, 6}$ & Telenor $(4)^{A}$ & PowerTech $(9)^{\mathrm{A}, 6}$ \\
\hline 2 & Universitetet i Oslo, NO (2) & Uninett $(1)^{\mathrm{X}, 6}$ & Broadnet $(10)^{\mathrm{A}}$ & PowerTech $(9)^{\mathrm{A}, 6}$ & - \\
\hline 3 & Høgskolen i Gjøvik, NO (3) & Uninett $(1)^{\mathrm{X}, 6}$ & PowerTech $(9)^{\mathrm{A}, 6}$ & - & - \\
\hline 4 & Universitetet i Troms $\varnothing$, NO (4) & Uninett $(1)^{X}$ & Telenor $(4)^{\mathrm{A}}$ & PowerTech $(9)^{\mathrm{A}, 6}$ & - \\
\hline 5 & Universitetet i Stavanger, NO (5) & Uninett $(1)^{\mathrm{X}}$ & Altibox $(11)^{6, F}$ & PowerTech $(9)^{\mathrm{A}, 6}$ & - \\
\hline 6 & Universitetet i Bergen, NO (6) & Uninett $(1)^{X, 6}$ & $\operatorname{BKK}(8)^{X, 6}$ & - & - \\
\hline 7 & Universitetet i Agder, NO (7) & Uninett $(1)^{\mathrm{X}, 6}$ & PowerTech $(9)^{\mathrm{A}, 6}$ & - & - \\
\hline 8 & Universitetet på Svalbard, NO (8) & Uninett $(1)^{\mathrm{X}}$ & Telenor $(4)^{\mathrm{F}}$ & - & - \\
\hline 9 & Universitetet i Trondheim, NO (9) & Uninett $(1)^{\mathrm{X}, 6}$ & PowerTech $(9)^{\mathrm{A}, 6}$ & - & - \\
\hline 10 & Høgskolen i Narvik, NO (10) & Uninett $(1)^{\mathrm{X}, 6}$ & Broadnet $(10)^{\mathrm{A}}$ & PowerTech $(9)^{A, 6}$ & - \\
\hline 11 & Høgskolen i Oslo og Akershus, NO (11) & Uninett $(1)^{\mathrm{X}, 6}$ & - & - & - \\
\hline 12 & Karlstads Universitet, SE (30) & SUNET $(20)^{X}$ & - & - & - \\
\hline 13 & Universität Kaiserslautern, DE (40) & DFN $(30)^{X, 6}$ & - & - & - \\
\hline 14 & Universität Duisburg-Essen, DE (42) & DFN $(30)^{X, 6}$ & Versatel $(31)^{\mathrm{A}}$ & - & - \\
\hline 15 & Hainan University, CN (88) & CERNET $(80)^{X}$ & China Unicom $(81)^{\mathrm{F}}$ & - & - \\
\hline 16 & The University of Kansas, US (100) & KanREN $(90)^{\mathrm{X}, 6}$ & - & - & - \\
\hline 17 & Bell Labs Seoul, KR (160) & KREONET $^{X, 6}$ & - & - & - \\
\hline
\end{tabular}

${ }^{6}$ With IPv6 support

$\mathrm{X}$ Business-grade fibre connection

${ }^{\mathrm{F}}$ Consumer-grade fibre connection

${ }^{\text {A }}$ Consumer-grade ADSL connection

Table I

The NorNet Core Sites IN JANUARY 2015

\begin{tabular}{|c|l|l|l|}
\hline No. & Full Name & Short Name & URL \\
\hline 1 & Uninett (1) & Uninett & https://www.uninett.no \\
\hline 2 & Hafslund (2) - now named Kvantel & Hafslund & http://kvantel.no \\
\hline 3 & Telenor Norge (4) & Telenor & https://www.telenor.no \\
\hline 4 & Bergenshalvøens Kommunale Kraftselskap (8) & BKK & http://bkk.no \\
\hline 5 & PowerTech (9) & PowerTech & http://www.powertech.no \\
\hline 6 & Broadnet (10) & Broadnet & https://www.broadnet.no \\
\hline 7 & Altibox (11) & Altibox & https://www.altibox.no \\
\hline 8 & Swedish University Network (20) & SUNET & http://www.sunet.se \\
\hline 9 & Deutsches Forschungsnetz (30) & DFN & https://www.dfn.de \\
\hline 10 & Versatel (31) & Versatel & http://www.versatel.de \\
\hline 11 & Korea Research Environment Open Network (70) & KREONET & http://www.kreonet.net \\
\hline 12 & China Education and Research Network (80) & CERNET & http://www.cernet.edu.cn \\
\hline 13 & China Unicom (81) & Unicom & http://www.chinaunicom.com \\
\hline 14 & Kansas Research and Education Network (90) & KanREN & http://www.kanren.net \\
\hline
\end{tabular}

Table II

THE NORNET CORE ISPS IN JANUARY 2015 
over Norway as well as locations abroad. The NORNET testbed consists of two parts:

- The wireless part NorNet EdGe [23] for 2G/3G/4G experiments, as well as

- The wired part NorNeT CORE [24], [25].

In this paper, we focus on NORNET CORE, i.e. on the wired part of the testbed.

\section{A. Sites and Internet Connectivity}

The structure of NORNET CORE is illustrated in Figure 1; Table I shows the site list ${ }^{3}$ and Table II the ISP list. Currently, NORNET CORE consists of 17 sites, with 11 sites in Norway, 2 sites in Germany as well as 1 site in China, Sweden, the U.S.A. and South Korea each. In total, 14 different ISPs are connected.

The NORNET CORE sites are hosted at universities and research organisations. Therefore, the local research network provider is the primary ISP of all sites (i.e. Uninett in Norway, SUNET in Sweden, DFN in Germany, CERNET in China and KanREN in Kansas, U.S.A.). While IPv4 support is of course available everywhere, IPv6 is available for most site setups; where not available, it is simply not deployed in the local network of the corresponding site yet.

Clearly, research networks are mostly overprovisioned. In order to see the effects of consumer-grade network accesses, a particular property of NORNET CORE is to offer such accesses as well. Therefore, the secondary ISPs use a mix of different access technologies, ranging from Asymmetric Digital Subscriber Line (ADSL), like e.g. PowerTech and Versatel, to consumer-grade fibre lines (like e.g. Altibox) and business-grade fibre connections (e.g. Hafslund and BKK). Whenever available from an ISP, IPv6 support is provided as well. However, unlike for the research network ISPs, getting IPv6 from commercial providers is still a challenge in many cases.

\section{B. Routing}

Routing in NORNET CORE [24], [25] is performed by a device denoted as tunnelbox, as illustrated in Figure 1. The tunnelbox is a Linux-based router that establishes a logical, fully-connected mesh of static IP tunnels among all NORNET CORE sites. Since the number of sites and ISPs is small, this structure is easily maintainable. For each ISP, a separate routing table is built. Based on the source address of a routed IP packet, the right table is selected by appropriate routing rules [26]. For example, if the source address is in the Uninett address range, the Uninett routing table is applied. This table then routes the packet out via Uninett. Alternatively, the outgoing ISP may also be selected by certain values [24] for the Type of Service/Traffic Class field of the IP packet. This gives the researcher control over the outgoing ISP. The destination address of course corresponds to the incoming ISP at the remote site.

Due to the tunnelling, it is possible to apply an own, systematic addressing scheme over the whole NORNET CORE testbed. For each site, the nodes use the following scheme:

\footnotetext{
${ }^{3}$ NORNET CORE Sites List: https://www.nntb.no/pub/nornet-configuration/ NorNetCore-Sites.html.
}

- IPv4: 10.Provider.Site.Node/24

- IPv6: 2001:700:4100:Provider|Site::Node/64

Provider and Site identify the ISP and the site (the corresponding values are provided in parentheses in Table I and Table II), while Node identifies a device.

Communication with the outside Internet is possible via routing through the central site at the Simula Research Laboratory for security reasons. For IPv4, due to the need for using a private address space, network/port address translation is necessary. The central site also hosts the management and monitoring infrastructure for the testbed (to be explained later).

Due to the heterogeneity of sites and connections, NORNET CORE covers a lot of very realistic Internet conditions that are relevant for research on multi-homed systems. For example, there are slow-speed links with buffer bloat (like ADSL [27]), long-distance connections (e.g. China to Norway [13]), very remote sites like Universitetet på Svalbard in Longyearbyen (just about 1,300 $\mathrm{km}$ from the North Pole), as well as a mix of research network and commercial ISP connections. Quality of service (QoS) characteristics of different paths can therefore vary significantly, and these conditions may change [10] over time (long-term as well as short-term). It is therefore highly useful for researchers to get real-time information about the paths, as well as to have a history of this information. Therefore, we have designed and developed the monitoring infrastructure that is described in the following.

\section{The MONITORING INFRASTRUCTURE}

Performance monitoring of servers in NORNET CORE is performed by using a combination of COLLECTD and GRAPHITE. Tunnelboxes are automatically configured to run Round-Trip Time (RTT) measurements by using the Internet Control Message Protocol (ICMP) across all tunnels present on the tunnelbox. Centralised configuration management is used to maintain configuration files and applications for all servers and tunnelboxes. The primary software deployed to visualise collected data are Horizon and GRAPHITE charts. RTT measurements from all tunnelboxes and all data collected by COLLECTD are stored in CARBON on the server perfmon1.simula.nornet ${ }^{4}$. They can be visualised or queried by using the GRAPHITE URL $\mathrm{API}^{5}$. In the following, we introduce all parts in detail.

\section{A. Configuration Management}

CFEnGINE is a widely used open source configuration management tool developed by Mark Burgess. It is known for its low resource consumption and flexible configuration language. In NorNet CORE, the CFEngine Community ${ }^{6}$ version is used to maintain global NORNET configuration files that need to be identical on all hosts. It is also used for running COLLECTD with various configurations and configuring the logstash-forwarder to ship system logs to a central log server: loghost 1 . simula. nornet.

CFEnGINE in concert with the NORNET CORE configuration database [24], [25] make sure that there is one COLLECTD

\footnotetext{
4 . nornet is the NORNET-internal, private top-level domain. The corresponding DNS infrastructure is available to all NORNET CORE users.

${ }^{5}$ GRAPHITE URL API: http://graphite.readthedocs.org/en/1.0/url-api.html.

${ }^{6}$ CFEnGINE Community: http://cfengine.com/community/.
} 
process running for each source address (IPv4 and IPv6) on all tunnelboxes. Each of these COLLECTD processes runs an instance of COLLECTD's ping plugin and is automatically configured to measure the ICMP RTT from that particular source address to all remote NORNET CORE tunnel endpoints.

\section{B. Visualising Data}

Due to the amount of tunnel endpoints, it is advisable to look at different subsets of metrics for the desired time period. Using the URL API of GRAPHITE, it is possible to query metrics by using globbing to select which subsets of metrics to visualise. Ordinary line charts quickly become overloaded, unless looking at a very low number of metrics at one time. Horizon charts ${ }^{7}$, however, use colour saturation combined with stacking, in order to reduce the vertical space for each single metric while still synchronising graphs time-wise. Horizon charts make it therefore possible to detect metric patterns over a larger metric subset than simple line charts. We will make its usefulness clearer with a full example in Section IV.

In NorNET CORE, Horizon charts are implemented to visualise subsets of tunnel ICMP RTT times. Our script uses Mike Bostock's ${ }^{8}$ Cubism JavasScript (CUBISMJS) ${ }^{9}$ combined with GRAPHITE metric globbing, in order to select and visualise subsets of RTT metrics. The resulting HTML page shows 15 hours of data, each one minute interval is represented by one pixel width horizontally (i.e. 900 pixels for 15 hours). The script retrieves parameters from the URL, allowing users to change the result to only show relevant data. In its current form, the script parameters support the selection of tunnel source and destination IP addresses in both, IPv4 and IPv6 formats. It is possible to visualise older measurement data by using a delay parameter, thus shifting the view backwards in time. The currently available parameters are:

- delayhours The desired time shift specified in hours (default: 0);

- dstip Destination IP as IPv4 or IPv6 address;

- srcip Source IP as IPv4 or IPv6 address;

- gheight The height of the Horizon chart in pixels (default: 50).

When using the dstip and srcip with IPv4 addresses, each dot in the address must be replaced by an underscore. The replacement is an artefact of GRAPHITE, which uses dots internally to separate between levels of metric hierarchy. IP tunnels in NORNET CORE are assigned addresses according to the system described in Subsection II-B, where certain parts of the IP address represent the site and the ISP identification numbers. By using this system, source and destination IP addresses together with globbing characters (i.e. the wildcard * for matching all, character ranges like [0-9] for matching the numbers 0 to 9 , or alternatives like $\{1,42\}$ in braces), can be used to retrieve metrics from particular sites and operators or combinations of them.

In the following, we provide two examples for the selection by utilising GRAPHITE globbing (the corresponding parameters are to be appended to the NorNet-

\footnotetext{
${ }^{7}$ Horizon charts: http://bl.ocks.org/mbostock/1483226

${ }^{8}$ Mike Bostock: http://bl.ocks.org/.

${ }^{9}$ CUBISMJS: https://square.github.io/cubism/.
}

internal URL http://perfmon1.simula.nornet/content/horizon/ nnc_tunnel_ping.html?):

a) Example 1: Uninett at Universitetet i Oslo (site index 2, see Table I) to all ISPs at the Simula Research Laboratory (site index 1, see Table I):

- IPv4: srcip=10_1_2_1\&dstip=10_* ${ }^{1}{ }^{1}{ }^{1}$

- IPv6: srcip=2001:700:4100:102::1\& dstip $=2001: 700: 4100: \star 01:$ :

b) Example 2: All ISPs on sites 2 to 9 and site 42 to PowerTech (provider index 9, see Table II) at Høgskolen i Gjøvik (site index 3, see Table I):

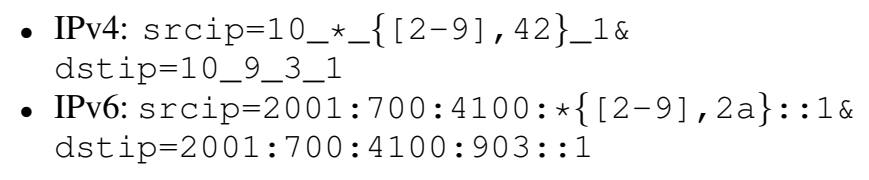

\section{Monitoring}

COLLECTD agents collect metrics locally on each NoRNET CORE host through their input plugins (CPU, memory, disk space, etc.) and send data to the server perfmon1. simula. nornet by using the COLLECTD network plugin. The network plugin acts as an output plugin for each of the machines in NORNET CORE and as an input plugin for perfmon1. simula. nornet. CARBON is the data storage and retrieval service in GRAPHITE. CARBON is running on perfmonl.simula.nornet and storing metric files (whisper files) on the host's local filesystem. COLLECTD on perfmon1.simula.nornet uses the CARBON writer output plugin to dispatch collected data from the NORNET CORE infrastructure into CARBON.

The COLLECTD configuration is controlled by CFENGINE, based on different server roles in NORNET CORE, but it is possible to add COLLECTD configuration by other means on individual NORNET CORE hosts by dropping additional files in /etc/collectd.d. All data COLLECTD is configured to collect ends up in the metric hierarchy of CARBON on perfmon1.simula.nornet, where it is made available for the NORNET CORE users. In the following, we provide a proof of concept that is e.g. very relevant for research on multi-path transport performance.

\section{RESUlts}

Figure 2 presents the results for the query of srcip $=10 \_*{ }^{*}{ }_{2}-1$ \&dstip $=10 \_*{ }^{*}{ }_{1}{ }_{1}$, obtaining the plots for the source site at Universitetet $\mathrm{i}$ Oslo (by filtering srcip=10_*_2_1, i.e. all ISPs and site index 2 - see Table I - for IPv4) and the destination site at the Simula Research Laboratory (by filtering dstip $=10_{-}{ }^{\star}{ }^{1}{ }_{-} 1$, i.e. all ISPs and site index 1 - see Table I - for IPv4). The height of the plots is 50 pixels (representing $50 \mathrm{~ms}$ per colour band, $200 \mathrm{~ms}$ alltogether), by using the default gheight setting. Since the site at Universitetet i Oslo has 3 ISPs (Uninett, Broadnet and PowerTech; see Table I) and the site at the Simula Research Laboratory has even 4 ISPs (Uninett, Hafslund, Telenor and PowerTech; see Table I), there are $3 * 4=12$ different source $\rightarrow$ destination combinations in total. In our setup, the tunnelbox at Universitetet i Oslo is named 

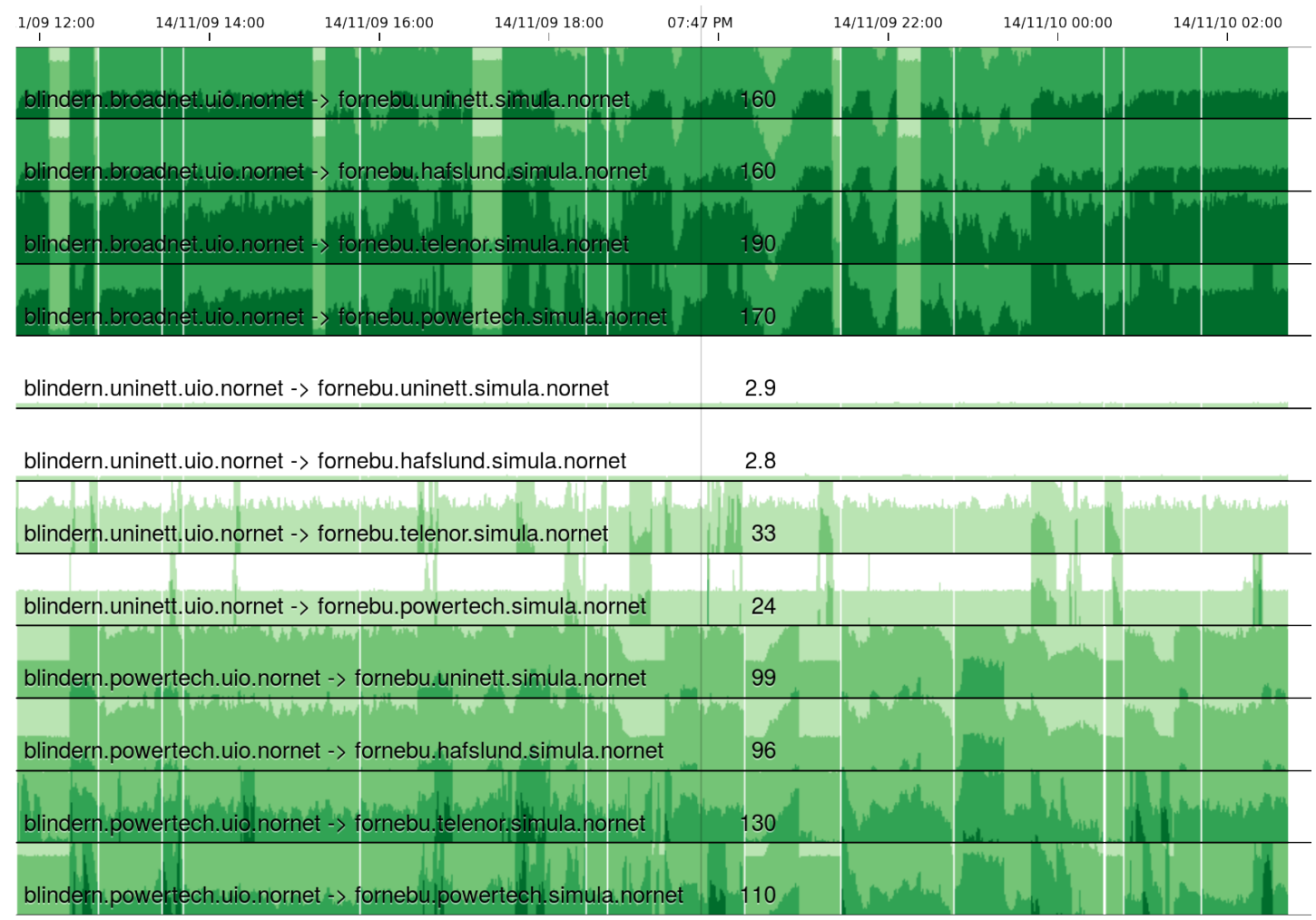

Figure 2. Results

blindern, while the tunnelbox at the Simula Research Laboratory is called fornebu ${ }^{10}$.

From the researcher's perspective, the two sites show a quite interesting behaviour: although the two sites are quite nearby (the bee line between the two setups is less than $10 \mathrm{~km}$ ), different combinations of ISPs have completely different QoS characteristics: The first four plots use Broadnet as outgoing ISP (blindern.broadnet.uio.nornet), which is an ADSL connection. Due to running experiments, there is some load on this ADSL interface, causing delay due to buffer bloat [28]. In the online version, the actual numerical values of the RTT for a certain time are shown by moving the mouse over the plots. Then, the times are displayed (here: at 07:47 PM, marked by a vertical line over all plots). With values between $160 \mathrm{~ms}$ and $190 \mathrm{~ms}$ here, an advantage of the Horizon plot becomes visible: since the RTT height of the plot is just $50 \mathrm{~ms}$, the plot "wraps around", i.e. it begins counting again from the bottom, but with a darkened colour. The darker the colour, the more wraps. That is, for a value of $190 \mathrm{~ms}$, there are 3 wraps (at $50 \mathrm{~ms}, 100 \mathrm{~ms}, 150 \mathrm{~ms}$ ), leading to a dark green line of $40 \mathrm{~ms}$ height. The resulting plot is therefore quite illustrative, while saving a significant amount of figure height. So, 12 plots (or even more) can be displayed easily on one screen page in landscape-format.

The Horizon plot's wrapping feature also becomes useful when comparing values to significantly different ones, here particularly for using Uninett as the outgoing ISP

\footnotetext{
${ }^{10}$ Blindern and Fornebu are the locations of the sites, hence the host names.
}

(blindern.uninett.uio.nornet - plots 5 to 8). Uninett is the Norwegian research network ISP, and provides a particularly low-latency connection between the two sites (there is a direct fibre link between both sites). The resulting RTT at 07:47 PM is therefore just $2.9 \mathrm{~ms}$. A similar value can be observed for Hafslund as the incoming ISP (fornebu.hafslund.simula.nornet) with $2.8 \mathrm{~ms}$. Obviously, sending to ADSL ISPs (Telenor and PowerTech, i.e. fornebu.telenor.simula.nornet and fornebu. powertech. simula. nornet) results in a significantly higher RTT (here: $33 \mathrm{~ms}$ and $24 \mathrm{~ms}$ at 07:47 PM). However, due to the wrapping feature of the Horizon chart, the values are easily comparable: mostly, they have the same light-green colour (representing the RTT value band from $0 \mathrm{~ms}$ to $50 \mathrm{~ms}$ ).

The last outgoing ISP at the Universitetet i Oslo is PowerTech (plots 9 to 12). However, unlike Broadnet in this setup, the RTT for this connection is significantly lower. This is directly visible by the lighter colours of the Horizon chart that represent lower RTT ranges. At 07:47 PM, the RTT ranges from $96 \mathrm{~ms}$ to $130 \mathrm{~ms}$, with less than $100 \mathrm{~ms}$ if the incoming ISP at the Simula Research Laboratory is a fibre connection (i.e. fornebu. uninett.simula. nornet or fornebu.hafslund.simula.nornet). So, with the results from the Horizon charts and the history database of the measurements, the researcher gets a very powerful tool for analysing the QoS characteristics during experiments and finally interpreting the results. Of course, the researcher can 
in addition also obtain the raw data for further processing.

Note, that the COLLECTD service on a tunnelbox checks for configuration updates about once per hour, at randomized intervals. Then, the RTT measurement service stops for a few seconds to update its configuration. This is visible by short intervals without RTT data. The current stop-update-restart mechanism is quite simple and going to be replaced by a more advanced strategy in the near future.

\section{CONCLUSiOnS}

NORNET CORE is becoming an increasingly popular platform for larger-scale Internet tests and evaluations of software that makes use of multi-homing. It provides developers and researchers the opportunity to easily deploy experiment software on multi-homed nodes that are distributed over a large geographical area, in multiple countries, with a variation of ISPs and access technologies and IPv4 as well as IPv6 support. However, it is important to get information on the QoS characteristics of the paths (i.e. ISP/site to ISP/site combinations) in the system, in order to debug and interpret observed effects in experiments. In this paper, we have therefore presented our maintenance and monitoring infrastructure for NORNET CORE that provides access to such information. Particularly, it does not only deliver up-to-date, real-time data but also stores historical information for time-series analyses.

As part of our future work, we are going to further refine the monitoring system, based on feedback from our NORNET CORE user community. The NORNET CORE testbed itself is going to be extended by some more sites outside of Norway, in order to further improve the research possibilities in longdistance and possibly inter-continental, multi-homed setups. Also, we will try to further increase the number of IPv6 endpoints. Our overall goal is to provide researchers the possibility to evaluate their concepts and approaches for multihomed systems in the real-world Internet, in order to finally bring their ideas to application by end-users.

\section{REFERENCES}

[1] P. Lei, L. Ong, M. Tüxen, and T. Dreibholz, "An Overview of Reliable Server Pooling Protocols," IETF, Informational RFC 5351, Sep. 2008, ISSN 2070-1721.

[2] T. Dreibholz, "Reliable Server Pooling - Evaluation, Optimization and Extension of a Novel IETF Architecture," Ph.D. dissertation, University of Duisburg-Essen, Faculty of Economics, Institute for Computer Science and Business Information Systems, Mar. 2007.

[3] R. R. Stewart, "Stream Control Transmission Protocol," IETF, RFC 4960, Sep. 2007, ISSN 2070-1721.

[4] P. D. Amer, M. Becke, T. Dreibholz, N. Ekiz, J. R. Iyengar, P. Natarajan, R. R. Stewart, and M. Tüxen, "Load Sharing for the Stream Control Transmission Protocol (SCTP)," IETF, Individual Submission, Internet Draft draft-tuexen-tsvwg-sctp-multipath-09, Oct. 2014.

[5] T. Dreibholz, "Evaluation and Optimisation of Multi-Path Transport using the Stream Control Transmission Protocol," Habilitation Treatise, University of Duisburg-Essen, Faculty of Economics, Institute for Computer Science and Business Information Systems, Mar. 2012.

[6] J. R. Iyengar, P. D. Amer, and R. R. Stewart, "Concurrent Multipath Transfer using SCTP Multihoming over Independent End-to-End Paths," IEEE/ACM Transactions on Networking, vol. 14, no. 5, pp. 951-964, Oct. 2006, ISSN 1063-6692.

[7] J. B. Postel, "Transmission Control Protocol," IETF, Standards Track RFC 793, Sep. 1981, ISSN 2070-1721.

[8] A. Ford, C. Raiciu, M. Handley, and O. Bonaventure, "TCP Extensions for Multipath Operation with Multiple Addresses," IETF, RFC 6824, Jan. 2013, ISSN 2070-1721.
[9] C. Raiciu, C. Paasch, S. Barré, A. Ford, M. Honda, F. Duchêne, O. Bonaventure, and M. Handley, "How Hard Can It Be? Designing and Implementing a Deployable Multipath TCP," in Proceedings of the 9th USENIX Conference on Networked Systems Design and Implementation (NSDI), San Jose, California/U.S.A., Apr. 2012, pp. 1-14.

[10] F. Golkar, T. Dreibholz, and A. Kvalbein, "Measuring and Comparing Internet Path Stability in IPv4 and IPv6," in Proceedings of the 5th IEEE International Conference on the Network of the Future (NoF), Paris/France, Dec. 2014, ISBN 978-1-4799-7531-0.

[11] A. Walid, J. Hwang, Q. Peng, and S. H. Low, "Balia (Balanced Linked Adaptation) - A New MPTCP Congestion Control Algorithm," in Proceedings of the 90th IETF Meeting, Toronto, Ontario/Canada, Jul. 2014.

[12] M. Becke, T. Dreibholz, H. Adhari, and E. P. Rathgeb, "On the Fairness of Transport Protocols in a Multi-Path Environment," in Proceedings of the IEEE International Conference on Communications (ICC), Ottawa, Ontario/Canada, Jun. 2012, pp. 2666-2672, ISBN 978-1-4577-2052-9.

[13] M. Becke, H. Adhari, E. P. Rathgeb, F. Fa, X. Yang, and X. Zhou, "Comparison of Multipath TCP and CMT-SCTP based on Intercontinental Measurements," in Proceedings of the IEEE Global Communications Conference (GLOBECOM), Atlanta, Georgia/U.S.A., Dec. 2013.

[14] S. Ferlin, T. Dreibholz, and Özgü Alay, "Multi-Path Transport over Heterogeneous Wireless Networks: Does it really pay off?" in Proceedings of the IEEE Global Communications Conference (GLOBECOM), Austin, Texas/U.S.A., Dec. 2014, pp. 5005-5011, ISBN 978-1-4799-3512-3.

[15] T. Dreibholz, X. Zhou, and F. Fa, "Multi-Path TCP in Real-World Setups - An Evaluation in the NorNet Core Testbed," in 5th International Workshop on Protocols and Applications with Multi-Homing Support (PAMS), Gwangju/South Korea, Mar. 2015.

[16] S. Ferlin, T. Dreibholz, and Özgü Alay, "Tackling the Challenge of Bufferbloat in Multi-Path Transport over Heterogeneous Wireless Networks," in Proceedings of the IEEE/ACM International Symposium on Quality of Service (IWQoS), Hong Kong/People's Republic of China, May 2014, pp. 123-128, ISBN 978-1-4799-4852-9.

[17] S. Ferlin, T. Dreibholz, Özgü Alay, and A. Kvalbein, "Measuring the QoS Characteristics of Operational 3G Mobile Broadband Networks," in Proceedings of the 4th International Workshop on Protocols and Applications with Multi-Homing Support (PAMS), Victoria, British Columbia/Canada, May 2014, pp. 753-758, ISBN 978-1-4799-2652-7.

[18] Øyvind Ytrehus, "Coding for Multipath TCP: Opportunities and Challenges," in Proceedings of the 2nd International NorNet Users Workshop (NNUW-2), Fornebu, Akershus/Norway, Aug. 2014

[19] D. A. Hayes, S. Ferlin, and M. Welzl, "Practical Passive Shared Bottleneck Detection using Shape Summary Statistics," in Proceedings of the 39th IEEE Conference on Local Computer Networks (LCN), Edmonton, Alberta/Canada, Sep. 2014, pp. 150-158.

[20] M. M. Yousaf, M. Welzl, and B. Yener, "On the Accurate Identification of Network Paths having a Common Bottleneck," in Proceedings of the ACM SIGCOMM Conference, Seattle, Washington/U.S.A., Aug. 2008, poster Presentation.

[21] A. Tveito, A. M. Bruaset, and O. Lysne, Simula Research Laboratory by thinking constantly about it. Heidelberg, Baden-Württemberg/Germany: Springer, Nov. 2009, ISBN 978-3642011559.

[22] T. Dreibholz, "The NorNet Testbed for Multi-Homed Systems - Introduction and Status," Invited Talk at Princeton University, Department of Computer Science, Princeton, New Jersey/U.S.A., May 2014.

[23] A. Kvalbein, D. Baltrūnas, K. R. Evensen, J. Xiang, A. Elmokashfi, and S. Ferlin, "The NorNet Edge Platform for Mobile Broadband Measurements," Computer Networks, Special Issue on Future Internet Testbeds, vol. 61, pp. 88-101, Mar. 2014, ISSN 1389-1286.

[24] E. G. Gran, T. Dreibholz, and A. Kvalbein, "NorNet Core - A MultiHomed Research Testbed," Computer Networks, Special Issue on Future Internet Testbeds, vol. 61, pp. 75-87, Mar. 2014, ISSN 1389-1286.

[25] T. Dreibholz and E. G. Gran, "Design and Implementation of the NorNet Core Research Testbed for Multi-Homed Systems," in Proceedings of the 3nd International Workshop on Protocols and Applications with MultiHoming Support (PAMS), Barcelona, Catalonia/Spain, Mar. 2013, pp. 1094-1100, ISBN 978-0-7695-4952-1.

[26] M. A. Brown, Guide to IP Layer Network Administration with Linux, Apr. 2003.

[27] H. Adhari, T. Dreibholz, M. Becke, E. P. Rathgeb, and M. Tüxen, "Evaluation of Concurrent Multipath Transfer over Dissimilar Paths," in Proceedings of the 1st International Workshop on Protocols and Applications with Multi-Homing Support (PAMS), Singapore, Mar. 2011, pp. 708-714, ISBN 978-0-7695-4338-3.

[28] V. Cerf, V. Jacobson, N. Weaver, and J. Gettys, "BufferBloat: What's Wrong with the Internet?" ACM Queue, vol. 9, no. 12, pp. 10-20, Dec. 2011, ISSN 1542-7730. 